found to harbour mutations in the isocitrate dehydrogenase 2 (IDH2) gene. The patient presented with an impalpable $8 \mathrm{~mm}$ right breast lesion, detected on staging CT scan for known endometrial carcinoma. The lesion was well defined and hypoechoic on ultrasound, and occult on mammogram. Core biopsy demonstrated a papillary neoplasm with focal atypical features. Excisional biopsy revealed a circumscribed papillary neoplasm composed of cuboidal to tall columnar cells with abundant granular eosinophilic cytoplasm. Nuclei were notably aligned away from the basal aspect of the cells and exhibited PTC-like nuclear features including grooves and intranuclear pseudo-inclusions. The cells diffusely expressed cytoplasmic CK5/6 and there was very focal nuclear positivity for ER. Calretinin was patchy and cytoplasmic. No intact myoepithelial layer was demonstrated. A diagnosis of SPCRP was made and ancillary molecular testing revealed a novel IDH2 variant c520G $>$ A, p.(Ala174Thr). SPCRP is a rare tumour with a favourable prognosis, which exhibits distinctive histological and immunophenotypical features that are readily recognisable to pathologists.

\section{UTERINE INFLAMMATORY MYOFIBROBLASTIC TUMOUR: A CASE REPORT}

Jordan Butler $^{1}$, Lyndal Anderson ${ }^{1}$, Diane Cominos ${ }^{2}$, Robert Rawson

${ }^{1}$ Department of Tissue Pathology and Diagnostic Oncology, NSW Health Pathology, Royal Prince Alfred Hospital, Sydney, Australia; and ${ }^{2}$ Histopathology and Cytopathology, Sullivan Nicolaides Pathology, Brisbane, Australia

Inflammatory myofibroblastic tumour (IMT) is a mesenchymal neoplasm with intermediate biological potential, which may be under-recognised in the uterus. ${ }^{1-3}$

Morphological and immunophenotypic overlap exists between uterine IMT and some other uterine mesenchymal neoplasms, including smooth muscle tumours. ${ }^{2}$ Morphological features suggestive of IMT may be subtle or focal and include myxoid change, tapered nuclei, and a lymphoplasmacytic infiltrate. ${ }^{1}$

Chromosomal rearrangements involving the anaplastic lymphoma kinase $(A L K)$ gene appear to be frequent in uterine IMT, ${ }^{1-3}$ and absent in other uterine mesenchymal neoplasms. ${ }^{2}$ Therefore, ALK immunohistochemistry (IHC) and $A L K$ florescent in situ hybridisation (FISH) appear to be useful tools to confirm the diagnosis of uterine IMT. ${ }^{3}$

Although most uterine IMTs follow a benign clinical course, local recurrence and metastases have been observed in a small subset. ${ }^{1}$ Correct diagnosis is important to ensure accurate prognosis and optimal management, which may include a tyrosine kinase inhibitor.

We present a case of uterine IMT with morphology and IHC suggestive of a leiomyosarcoma with myxoid change. ALK IHC was positive and an $A L K$ rearrangement was demonstrated with FISH, confirming the diagnosis of uterine IMT.

This case illustrates the importance of a low threshold for ALK testing in uterine mesenchymal tumours with morphological features suggestive of IMT.

\section{References}

1. Pickett JL, Chou A, Andrici JA, et al. Inflammatory myofibroblastic tumors of the female genital tract are under-recognized. Am J Surg Pathol 2017; 41: 1433-42.

2. Mohammad N, Haimes JD, Mishkin S, et al. ALK is a specific diagnostic marker for inflammatory myofibroblastic tumor of the uterus. Am J Surg Pathol 2018; 42: 1353-9.
3. Parra-Herran C, Quick CM, Howitt BE, et al. Inflammatory myofibroblastic tumor of the uterus: clinical and pathologic review of 10 cases including a subset with aggressive clinical course. Am J Surg Pathol 2015; 39: 157-68.

\section{LOCALISED PULMONARY CRYSTAL-STORING HISTIOCYTOSIS WITH UNDERLYING EXTRANODAL MARGINAL ZONE LYMPHOMA: A CASE REPORT}

Sunisha Chahal, J. J. Serfontein, Nicola Kingston

Department of Anatomical Pathology, LabPLUS, Auckland City Hospital, Auckland, New Zealand

Crystal-storing histiocytosis (CSH) is a rare entity, composed of a proliferation of non-neoplastic histiocytes with an accumulation of intracytoplasmic crystalline material. It is often associated with an underlying lymphoproliferative disorder, most often those that express monoclonal immunoglobulins, such as multiple myeloma and lymphoplasmacytic lymphoma. Here we report a case of localised pulmonary $\mathrm{CSH}$ in an asymptomatic 54-year-old man, found to have an incidental solid lesion in the right lung on computed tomography (CT). The lesion was composed of sheets of CD68 positive histiocytes that contained refractile eosinophilic crystalline inclusions, scattered lymphoid follicles of CD20 positive B lymphocytes, and occasional plasma cells. PCR for immunoglobulin heavy chain gene rearrangement confirmed the presence of a monoclonal population of B cells. These findings were supportive of a diagnosis of CSH with an underlying B cell/ plasma cell lymphoproliferative disorder, which was favoured to be an extranodal marginal zone lymphoma after clinical and radiological workup. Recognition of this rare entity is important as it may obscure an underlying lymphoproliferative disorder. A diagnosis of CSH should prompt careful exclusion of neoplasia, including clinical workup for systemic disease. In this case report we also discuss clinical features and diagnostic pitfalls.

\section{WHOLE EXOME SEQUENCING (WES) ENHANCES THE DIAGNOSTIC RATE OF PERINATAL AUTOPSY: A PROSPECTIVE CLINICAL UTILITY TRIAL WITH IMPLICATIONS FOR PRENATAL DIAGNOSIS}

Fiona Chan $^{1,2,3}$, Alison Yeung ${ }^{4,5}$, Anand Vasudevan ${ }^{2,4}$, Zornitza Stark ${ }^{4,6}$, Stacey Prystupa ${ }^{5}$, Yuen $\mathrm{Chan}^{5}$, Trishe Leong ${ }^{7}$, Kerryn Ireland-Jenkin ${ }^{7,8,9}$, Susan Fawcett ${ }^{2}$, Melissa Graetz ${ }^{8}$, Katherine Rose ${ }^{5}$, Samantha Ayres ${ }^{3,4}$, Anna Jarmolowicz ${ }^{3,4}$, Gemma Brett ${ }^{4,6}$, Yael Prawer ${ }^{3,5}$, Heather Chalinor ${ }^{7}$, Candice Dao ${ }^{8}$, Tenielle Davis ${ }^{2}$, Lisa Hui ${ }^{9}$, Mark Teoh ${ }^{5}$, Shelley Rowlands ${ }^{2}$, Susan Walker ${ }^{8}$, Elly Lynch $^{3,10}$, Melissa Martyn ${ }^{3,10}$, Belinda Chong, ${ }^{4,6}$, Clara Gaff ${ }^{3,10}$, Sebastian Lunke ${ }^{4,6}$, Jackie Collett ${ }^{1,2}$, George McGillivray ${ }^{2,4}$

${ }^{1}$ Royal Children's Hospital, Melbourne, Australia; ${ }^{2}$ Royal Women's Hospital, Melbourne, Australia; ${ }^{3}$ Melbourne Genomic Health Alliance, Melbourne, Australia; ${ }^{4}$ Murdoch Children's Research Institute, Melbourne, Australia; ${ }^{5}$ Monash Medical Centre, Melbourne, Australia; ${ }^{6}$ Victorian Clinical Genetics Services, Melbourne, Australia; ${ }^{7}$ Austin Health, Melbourne, Australia; ${ }^{8}$ Mercy Hospital for Women, Melbourne, Australia; ${ }^{9}$ University of Melbourne, Melbourne, Australia; and ${ }^{10}$ Walter and Eliza Hall Institute, Melbourne, Australia 
Genomic classification is rapidly becoming a routine and integral part of diagnosis in pathology. Perinatal pathology is following this trend. The aims of this study were to determine the utility of WES in perinatal autopsy for congenital anomalies and to model the outcome of WES as a prenatal test. A total of 131 probands with congenital anomalies who underwent post mortem examination were referred by pathologists to the study. 82 probands were considered suitable for sequencing. The parents of 5 declined enrolment and 10 could not be consented. 67 probands were enrolled. Autopsy identified specific diagnoses in 11 cases (17\%). WES identified specific diagnoses ('pathogenic' or 'likely pathogenic' variants) in 23 cases - a diagnostic rate of $35 \%$. The combined diagnostic rate of autopsy and sequencing was $38 \%$. A geneticist blinded to the autopsy findings reviewed the probands' antenatal imaging reports and recommended a gene list to model the clinical utility of prenatal WES. The use of antenatal sequencing in this cohort would have identified a specific diagnosis in 18 of the 23 cases with positive sequencing findings. In conclusion, WES doubles the diagnostic rate of autopsy for congenital anomalies and our data supports the prenatal use of genomic sequencing.

\section{PRACTICE FOR SPEED AND PROFICIENCY IN CYTOLOGY EXAMS USING THE NEW RCPA DIGITAL CYTOLOGY ECASES AND SECTRA}

Amanda Charlton ${ }^{1}$, Simone L. Van Es ${ }^{2}$

${ }^{1}$ Department of Histopathology, LabPlus, Auckland District Health Board, Auckland, New Zealand; and ${ }^{2}$ Department of Pathology, School of Medical Sciences, UNSW, Sydney, NSW, Australia

Aim: Create and share a digital a slide workshop using Sectra slides.

Background: The RCPA Cytopathology exam is planned to transition to digital slides viewed on the Sectra platform. Screening and reaching a diagnosis using digital cytology is slower than glass slides. ${ }^{1}$ Proficiency and speed comes with practice. ${ }^{2}$ There are currently $>200$ cases of digital cytology in the RCPA eCases library, many with $\mathrm{z}$-axis focus and digital annotations. Registrars and supervisors need to know how to efficiently navigate and share digital practice slide sets.

Methods: A metaphor of door, library and window help us navigate the data architecture. Enter the cytology slide library through the DOOR of the RCPA website. The cytology slides are found in the eCases LIBRARY, a digital repository of virtual slides linked to case data and diagnosis. View and share the slides via the Sectra WINDOW. The methods are demonstrated in 6 online videos. ${ }^{3}$

Result: Follow these steps to make and share a Sectra digital slide set. There is also an online example test set (https:// digipathed.wordpress.com/2020/01/07/sectra-practice-test-sets/) and an answer set (https://digipathed.wordpress.com/2020/01/ 07/sectra-practice-test-set-with-answers/).

Conclusion: Teaching and learning with digital slide sets is an opportunity to practice digital proficiency, which is a 21 st century skill for all of us.

\section{References}

1. House JC, Henderson-Jackson EB, Johnson JO, et al. Diagnostic digital cytopathology: Are we ready yet? J Pathol Informatics 2013 Oct; 4(28)

2. Van Es SL. Digital pathology: semper ad meliora. Pathology 2019; 51: $1-10$.
3. Charlton A. Practice for speed and proficiency in cytology exams using the new RCPA digital cytology eCases and Sectra. 6 videos. Cited 20 Jan 2020. https://digipathed.wordpress.com/2020/01/02/ prepare-for-the-rcpa-digital-cytology-exam-using-sectra/

\section{WHY DOES MY BRAIN HURT? PATHOLOGIST PERFORMANCE AND PATTERN RECOGNITION}

Amanda Charlton $^{1}$, Ann Carrigan ${ }^{2}$, Andrew Georgiou ${ }^{3}$, ${\text { Kim } \text { Curby }^{2} \text {, Thomas J. Palmeri }}^{4}$, Mark W. Wiggins ${ }^{2}$

${ }^{1}$ Department of Histopathology, Auckland City Hospital, and Department of Molecular Medicine and Pathology, University of Auckland, New Zealand; ${ }^{2}$ Department of Psychology, Macquarie University, Sydney, Australia; ${ }^{3}$ Centre for Health Systems and Safety Research, Macquarie University, Sydney, Australia; and ${ }^{4}$ Department of Psychology, Vanderbilt University, Nashville, TN, United States

Aim: To test whether indicators of pattern recognition moderate performance of new tasks.

Background: You, or your colleagues may have participated in this research, where we recruited Anatomical Pathologists and Registrars at the 2019 RCPA Update meeting, and online, to complete an online series of tasks.

Methods: We recruited 54 participants, who completed a series of 5 online tasks. ${ }^{1}$ To measure 'brain strain', 27 participants wore an infrared head band measuring pre-frontal cortex blood flow. ${ }^{2}$ Result: Higher pattern recognition was associated with greater accuracy on diagnostic tasks, and diagnosis of uncommon entities. Those participants with lower pattern recognition were less likely to select unlisted diagnoses for example, 'none of the above' in multiple choice questions. High pattern recognition was associated with higher cerebral blood flow.

Conclusion: The results validated our online task tool. ${ }^{1}$ The finding that those participants with lower pattern recognition skills were more likely to select an incorrect diagnosis from a list of answers indicates susceptibility to confirmation, and availability biases. ${ }^{3}$ Those participants with higher pattern recognition were less prone these cognitive errors. The finding of higher 'brain strain' in those with high pattern recognition suggests that higher levels of accuracy were achieved with greater cognitive effort. Reproducibility and longitudinal studies are planned.

\section{References}

1. Wiggins MW, Harris J, Loveday T, et al. EXPERTise (Software Package). Sydney: Macquarie University, 2010.

2. Unni A, Ihme K, Surm H, et al. Brain activity measured with fNIRS for the prediction of cognitive workload. 6th IEEE International Conference on Cognitive Infocommunications (CogInfoCom) 2015; 349-54.

3. Kahneman D. Thinking, Fast and Slow. New York: Farrar, Straus and Giroux, 2011.

\section{ADULT MESENCHYMAL HAMARTOMA OF LIVER: A CASE REPORT AND DISCUSSION OF ASSOCIATED MOLECULAR ABNORMALITIES}

Kimberley Chung, Chris Van Vliet, Bastiaan de Boer PathWest Laboratory Medicine, QEII Medical Centre and Fiona Stanley Hospital, Perth, WA, Australia

Mesenchymal hamartoma of liver (MHL) is the third most common paediatric liver tumour. It accounts for $8 \%$ of all liver 